\title{
Current new challenges in the management of ulcerative colitis
}

\author{
Tomohiro Fukuda, Makoto Naganuma, Takanori Kanai \\ Division of Gastroenterology and Hepatology, Department of Internal Medicine, Keio University School of Medicine, Tokyo, Japan
}

Ulcerative colitis (UC) is a chronic inflammatory condition of the gastrointestinal tract. Although the cause of UC is postulated to be multifactorial in nature, including genetic predisposition, epithelial barrier defects, dysregulation of immune responses, and environmental factors, the specific pathogenesis of UC is still incompletely understood. In the treatment of UC so far, a method of suppressing immunity and treating it has been mainstream. Immunosuppressant drugs, including thiopurines (azathioprine or 6-mercaptopurine), anti-tumor necrosis factor- $\alpha$ (anti-TNF- $\alpha$ ) antibody (infliximab and adalimumab), and calcineurin inhibitor, can be used in treat patients with corticosteroid-dependent and/or corticosteroid-refractory moderateto-severe UC. Recently, in addition to such a conventional therapeutic agent, golimumab, which is the first transgenic human monoclonal anti-TNF- $\alpha$ antibody to be fabricated, anti $\alpha-4 / \beta-7$ integrin antibody, and Janus kinase inhibitor have been reported to novel immunosuppressant therapy. Furthermore, other treatments with unique mechanisms different from immunosuppression, have also been suggested, including fecal microbiota transplantation and Indigo naturalis, which is a Chinese herbal medicine. We compared the features and efficacy of these new treatments. In this issue, the features and treatment options for these new treatments is reviewed. (Intest Res 2019;17:36-44)

Key Words: Colitis, ulcerative; Vedolizumab; Tofacitinib; Indigo naturalis; Fecal microbiota transplantation

\section{INTRODUCTION}

Ulcerative colitis (UC) is a chronic inflammatory condition of the GI tract, which is associated with diarrhea, bloody stool, and abdominal pain. ${ }^{1}$ Although the cause of UC is postulated to be multifactorial in nature, including genetic predisposition, epithelial barrier defects, dysregulation of immune responses, and environmental factors, the specific pathogenesis of UC is still incompletely understood.

For patients with mild-to-moderate UC, use at oral and topical 5-aminosalicylates (5-ASA) are recommended as a first-line

Received September 11, 2018. Revised October 5, 2018.

Accepted October 12, 2018.

Correspondence to Takanori Kanai, Division of Gastroenterology and Hepatology, Department of Internal Medicine, Keio University School of

Medicine, 35 Shinanomachi, Shinjuku-ku, Tokyo 160-8582, Japan.

Tel: +81-3-5843-7090, Fax: +81-3-5843-7091, E-mail: takagast@z2.keio.jp

ORCID Tomohiro Fukuda (https://orcid.org/0000-0002-6539-5002), Takanori

Kanai (https://orcid.org/0000-0002-1466-4532) therapy. Patients who do not achieve remission with 5-ASA drugs should be treated with corticosteroids. ${ }^{1}$ Although efficacy at 30 days after corticosteroid administration in UC patients was $80 \%$, only $49 \%$ maintained clinical response for 1 year, and $22 \%$ was steroid dependency. ${ }^{2}$ Immunosuppressant drugs, including thiopurines (azathioprine or 6-mercaptopurine), antiTNF- $\alpha$ antibody, and calcineurin inhibitor, can be used to treat patients with steroid dependency and/or steroid refractory moderate-to-severe UC. Recently, golimumab (GLM), which is the first transgenic human monoclonal anti-TNF- $\alpha$ antibody to be fabricated, anti $\alpha-4 / \beta-7$ integrin antibody, and Janus kinase (JAK) inhibitor have been reported to novel immunosuppressant therapy. Other treatments with unique mechanisms different from immunosuppression, have also been suggested, including fecal microbiota transplantation (FMT), which improves intestinal microbiota, and Indigo naturalis, which is a Chinese herbal medicine. Our aim in this review was to summarize current evidence and mechanisms of therapeutic effec- 
tiveness for current UC therapies.

\section{ANTI-TNF- $\alpha$ ANTIBODY}

Anti-TNF- $\alpha$ antibody is key drug to induce and maintenance remission in patients with moderate-to-severe UC. TNF- $\alpha$ is an inflammatory cytokine, being present either as a membrane protein of cells or in soluble form in blood. ${ }^{3}$ Anti-TNF- $\alpha$ antibody neutralizes soluble TNF- $\alpha$ and binds membranebound TNF- $\alpha$ to prevent cell damage, Anti-TNF- $\alpha$ antibody also suppresses TNF- $\alpha$ expression by inducing apoptosis of TNF- $\alpha$ producing cells. Since August 2018, anti-TNF- $\alpha$ antibody such as infliximab (IFX), adalimumab (ADA), and GLM, have been available for the treatment of UC, with demonstrated therapeutic efficacy of these drugs in Japan. ${ }^{4-7}$ However, a head-to-head comparison of these 3 different drugs has yet to be completed to determine any superiority. However, loss of response (LOR) is an important limitation of these drugs. As an example, among patients with CD treated using anti-TNF- $\alpha$ antibody, LOR develops in about $37 \%$ of cases, with $13 \%$ of these patients being unable to continue with anti-TNF- $\alpha$ antibody for up to 1 year. The mean percentage of patients with LOR of IFX was $37 \%$ and the annual risk for LOR of IFX was calculated to be $13 \%$ per patient-year. ${ }^{8}$ Thus, many patients on anti-TNF- $\alpha$ antibody therapy will need to change to another treatment for UC at some point over the course of their treatment.

A low trough level of anti-TNF- $\alpha$ antibody and anti-drug (anti-TNF- $\alpha$ antibody) antibody (ATA) have been identified as causes of LOR. ${ }^{9}$ There are many reports that optimization of treatment by trough concentration and ATA (treat to target) is effective. ${ }^{10}$ For example, Vande Casteele et al. ${ }^{11}$ reported that targeting IFX trough level to $3-7 \mathrm{mg} / \mathrm{mL}$ by optimizing dose and interval, results in a more efficient use of the drug. Furthermore, after dose optimization, continued concentrationbased dosing was associated with fewer disease relapse during the course of treatment. Although it is a retrospective study, efficacy by increasing the dose of anti-TNF- $\alpha$ antibody in UC has also been reported. ${ }^{12}$ However, trough level or ATA cannot be measured in many countries including Japan. In this case, anti-TNF- $\alpha$ antibody is optimized using other biomarkers. Although it is research on CD, CALM trial reported that the proportion of patients with Mucosal healing and steroid free at 48 weeks after entry was higher with early optimization of ADA using biomarkers such as serum CRP and fecal calprotectin. ${ }^{13}$

ATA inhibits the binding of anti-TNF- $\alpha$ antibody to the TNF- $\alpha$ molecule, with the resulting immune complex formation increasing the clearance rate of anti-TNF- $\alpha$ antibody which, ultimately, lowers the blood concentration of anti-TNF- $\alpha$ antibody. ${ }^{14,15}$ The rate of ATA production among patients with CD after having achieved remission with a $5 \mathrm{mg} / \mathrm{kg}$ dose of IFX was evaluated for different doses of maintenance IFX, 5 and $10 \mathrm{mg} / \mathrm{kg}$ and compared with a placebo. ${ }^{16}$ The rate of ATA production was $10 \%$ at an IFX dose of $5 \mathrm{mg} / \mathrm{kg}$ and $7 \%$ at a dose of $10 \mathrm{mg} / \mathrm{kg}$, compared to $30 \%$ in the placebo group. Therefore, the discontinuation of anti-TNF- $\alpha$ antibody therapy may increase the incidence of ATA production and may attenuate the effectiveness of anti-TNF- $\alpha$ antibody therapy when resumed. There is also evidence regarding additional therapeutic effectiveness of combination therapy of immunomodulator (IM) together and IFX to suppress ATA production, as reported in the UC-SUCCESS trial. ${ }^{17}$ The efficacy of combining IM with either ADA or GLM remains to be determined. Among anti-TNF- $\alpha$ antibody drugs available, GLM, which is synthesized using a transgenic method, might have a lower rate of ATA production than either IFX or ATA. However, a prospective trial for direct comparison of ATA production and rate of LOR for these 3 anti-TNF- $\alpha$ antibody drugs is needed.

Overall, there is currently no clear evidence to distinguish the selection of IFX, ADA or GLM at this time, with selection being based on clinical criteria, such as route of administration, administration time and frequency of scheduled visits for treatment (Table 1).

Table 1. Difference in Anti-TNF- $\alpha$ Antibodies

\begin{tabular}{lccc}
\hline Item & Infliximab & Adalimumab & Golimumab \\
\hline Administration interval (induction phase) & At wk 0, 2, & Every 2 wk & At wk 0, 2, 6 \\
Administration interval (maintenance phase) & Every 8 wk & Every 2 wk & Every 4 wk \\
Administration route & Intravenous administration & SC injection & SC injection \\
Self-administration & $\times$ & 0 & $\times$ \\
Efficacy of combination therapy of immunomodulator & 0 & Unclear & Unclear \\
\hline
\end{tabular}




\section{VEDOLIZUMAB}

Integrins are obligatory type I heterodimers that include $\alpha$ and $\beta$ subunits and are found on the surface of cells, such as leucocytes. In humans, 18 types of $\alpha$-subunits and $8 \beta$-subunits have been identified, yielding 24 different types of integrins comprised of different combinations of $\alpha$ and $\beta$-subunits. Integrins bind specifically on sites of adhesion which are expressed on vascular endothelial cells, and allow T lymphocytes to migrate into intestinal tissue, where they induce inflammation. ${ }^{18}$

Vedolizumab binds to $\alpha 4 \beta 7$ integrin on the surface of inflammatory cells, suppressing the migration of $\mathrm{T}$ lymphocytes into intestinal tissue, producing an anti-inflammatory effect. Specifically, the $\alpha 4 \beta 7$ integrin is a cell-surface glycoprotein that is expressed on $\mathrm{T}$ lymphocytes and induces inflammation through its interaction with the mucosal addressin cell adhesion molecule 1 (MAdCAM-1) on intestinal vascular endothelial cells. Because MAdCAM- 1 is selectively expressed in the vascular endothelium of the intestinal tract, the action of vedolizumab is probably confined to the intestinal tract.

The efficacy and safety of vedolizumab for induction and maintenance therapy of UC has been reported in the GEMINI-I trial. ${ }^{19}$ The GEMINI-I trial is a phase 3, randomized, double-blind, placebo-controlled study of 374 patients with moderate-to-severe UC. To evaluate the effectiveness of vedolizumab for induction of UC remission, patients were randomly assigned to receive intravenous vedolizumab (300 $\mathrm{mg}$ ) or a placebo, on days 1 and 15. Based on these definitions, the clinical rate of response to vedolizumab was $47.1 \%$, which was higher than the $25.5 \%$ rate in the placebo group $(P<0.001)$.

To evaluate the effectiveness of vedolizumab for maintenance therapy, patients who achieved a clinical response at week 6 were randomly assigned to one of the following groups for maintenance therapy: vedolizumab every 8 weeks (with placebo administered every other visit to preserve blinding); vedolizumab every 4 weeks; or placebo. The prescribed treat- ment was continued for 52 weeks, with the primary outcome being clinical remission, defined as a Mayo Clinic score $\leq 2$ (with no subscore higher than 1) and mucosal healing, defined as an endoscopic subscore of 0 or 1 . The rate of clinical remission at 52 weeks was $41.8 \%$ for the group receiving vedolizumab every 8 weeks, $44.8 \%$ for the group receiving vedolizumab every 4 weeks and $15.9 \%$ for the placebo group. Therefore, the rate of clinical remission was higher among patients receiving vedolizumab than the placebo group $(P<0.001)$. No difference in the rate of adverse events was identified in the GEMINI-I trial between the vedolizumab and placebo group (Table 2).

Systematic review showed that clinical response and remission were achieved in $43 \%$ and $25 \%$ by week 6 , respectively, and in $51 \%$ and $30 \%$ by week 14 to 22 . At week 52 , clinical response and remission were achieved in $48 \%$ and $39 \%$ of the patients, respectively. Adverse effects were mostly minor and occurred in $30.6 \%$ of the patients. ${ }^{20}$ Since the approval of vedolizumab, several real-world experience studies were reported. For example, Kopylov et al. ${ }^{21}$ reported that clinical response at week 14 after Vedolizumab administration was $23.5 \%$, at week 52 was $45 \%$. Amiot et al. ${ }^{22}$ also showed that the effect of Vedolizumab for UC was better at 14 weeks than at 6 weeks. These results suggested that the effectiveness of Vedolizumab appears slowly in some cases. There are also reports that the use of calcineurin inhibitor and vedolizumab together showed that $64 \%$ clinical response was obtained for 52 weeks after the end of calcineurin inhibitor. ${ }^{23}$ Namely, because the onset of effect is slow but safety is high, the effectiveness for maintenance therapy after introduction of remission is suggested.

Of note, the use of natalizumab, integrin $\alpha 4$ inhibitor, was associated with progressive multifocal leukoencephalopathy (PML) in clinical trials due to the reactivation of the JC polyoma virus. This was not an issue in the GEMINI-I trial. Moreover, as vedolizumab acts locally on the intestinal tract, the risk of systemic side effects is likely to be quite low. Currently, the long-term safety of vedolizumab is continuing to be evalu-

Table 2. Efficacy of Vedolizumab in GEMINI Trials

\begin{tabular}{|c|c|c|}
\hline Treatment phase & Primary outcome & Results \\
\hline Induction & Clinical response at wk $6^{a}$ & $47.1 \%, 25.5 \%$ (receive intravenous vedolizumab or placebo at day 1 and 15 ), $P<0.001$ \\
\hline Maintenance & Clinical remission at wk $52^{b}$ & $\begin{array}{l}\text { 44.8\%, 41.8\%, 15.9\% (300 mg/4 wk, } 300 \text { mg/8 wk, placebo), } 300 \text { mg/4 wk vs. placebo }(P<0.001) \text {, } \\
300 \text { mg/8 wk vs. placebo }(P<0.001)\end{array}$ \\
\hline
\end{tabular}

${ }^{a}$ Clinical response defined as a reduction in the Mayo Clinic score of at least 3 points and a decrease of at least 30\% from the baseline score, with a decrease of at least 1 point on the rectal bleeding subscale or an absolute rectal bleeding score of 0 or 1 .

${ }^{b}$ Clinical remission defined as a Mayo Clinic score of 2 or lower and no subscore higher than 1, and mucosal healing, defined as an endoscopic subscore of 0 or 1. 
ated in the GEMINI long-term safety (LTS) study. ${ }^{24}$

\section{TOFACITINIB}

JAK refers to 4 intracellular types of tyrosine kinase (JAK1, JAK2, JAK3, and tyrosine kinase 2) that activate signal transducers and activators of transcription (STATs). Inflammatory cytokines bind to JAK receptors on cell membranes. As JAK receptors are phosphorylated, they are activated by this binding. The transcription factor STAT is attracted to the phosphorylated JAK receptor and activated. The activated STAT forms a dimer and migrates inside the cell nucleus, initiating gene transcription, and ultimately, the action of the cytokine is exerted. The combination of the 4 types of JAK and 7 types of STAT causes various effects. ${ }^{25}$ Tofacitinib inhibits all JAKs, with a more specific and strong inhibitory effect on JAK types 1 and 3. As such, tofacitinib suppresses inflammation via its inhibition of the JAK-STAT system.

The OCTAVE trial provided evidence of the efficacy of tofacitinib in inducing and maintaining disease remission among patients with UC. ${ }^{26}$ The OCTAVE induction 1 trial included 598 patients with moderate-to-severe UC. Patients were randomly assigned to the tofacitinib ( $10 \mathrm{mg}$ twice daily for 8 weeks) or placebo group. The primary outcome was clinical remission at 8 weeks, defined by a total Mayo score of $\leq 2$, with no subscore $>1$, and a rectal bleeding subscore of 0 . The rate of remission induction was $18.5 \%$ in the tofacitinib group, compared to $8.2 \%$ in the placebo group $(P=0.007 ; 95 \% \mathrm{CI}, 4.3-16.3)$. The OCTAVE induction 2 trial was conducted with 541 patients, again with moderate-to-severe UC, to verify the outcomes of the first phase, using the same criteria for clinical remission. In the OCTAVE induction 2 trial, the rate of remission induction was $16.6 \%$ in the tofacitinib group, compared to $3.6 \%$ in the placebo group ( $P<0.001 ;$ 95\% CI, 8.1-17.9) (Table 3 ).

To evaluate the efficacy of tofacitinib to maintain disease remission, 593 patients who had achieved a clinical response at 8 weeks in the induction trial, were randomly assigned to the following 3 groups for 52 weeks: oral dose of tofacitinib of 5 $\mathrm{mg}$ twice daily, oral dose of $10 \mathrm{mg}$ twice daily and a placebo. In the maintenance phase of the OCTAVE trial. The remission rate at 52 weeks was $34.3 \%$ in the 5 -mg tofacitinib group and $40.6 \%$ in the 10 -mg tofacitinib group, compared to $11.1 \%$ in the placebo group $(P<0.001)$ (Table 3$)$.

Infection is the main side effect of tofacitinib, in the OCTAVE induction $1 / 2$ trial, with an incidence rate of $23.3 \%$ and $18.2 \%$ for the 10-mg tofacitinib groups, compared to $15.6 \%$ and $15.2 \%$
Table 3. Efficacy of Tofacitinib in OCTAVE Trials

\begin{tabular}{|c|c|c|}
\hline Trial & $\begin{array}{l}\text { Primary } \\
\text { outcome }\end{array}$ & Results \\
\hline $\begin{array}{l}\text { OCTAVE induction } \\
1 \text { (induction) }\end{array}$ & $\begin{array}{l}\text { Remission at } \\
8 w^{a}\end{array}$ & $\begin{array}{l}18.5 \%, 8.2 \% \text { (10 mg, placebo), } \\
P=0.007\end{array}$ \\
\hline $\begin{array}{l}\text { OCTAVE induction } \\
2 \text { (induction) }\end{array}$ & $\begin{array}{l}\text { Remission at } \\
8 w^{a}\end{array}$ & $\begin{array}{l}16.6 \%, 3.6 \% \text { (10 mg, placebo), } \\
P<0.001\end{array}$ \\
\hline $\begin{array}{l}\text { OCTAVE sustain } \\
\text { (maintenance) }\end{array}$ & $\begin{array}{l}\text { Remission at } \\
52 w^{a}\end{array}$ & $\begin{array}{l}34.3 \%, 40.6 \%, 11.1 \% \text { ( } 5 \mathrm{mg} / \mathrm{BID}, \\
10 \mathrm{mg} / \mathrm{BID} \text {, placebo), } \\
5 \mathrm{mg} / \mathrm{BID} \text { vs. placebo }(P<0.001) \\
10 \mathrm{mg} / \mathrm{BID} \text { vs. placebo }(P<0.001)\end{array}$ \\
\hline
\end{tabular}

${ }^{a}$ Remission defined as a total Mayo score of $\leq 2$, with no subscore $>1$ and a rectal bleeding subscore of 0 .

in the placebo group. In the OCTAVE sustain trial, the infection rate was $35.9 \%$ in the 5 -mg tofacitinib group, $39.8 \%$ in the 10-mg tofacitinib group and $24.2 \%$ in the placebo group. Especially, a Herpes zoster virus infection occurred in $1.5 \%$ of infection cases in the 5 -mg group, $5.1 \%$ in the $10-\mathrm{mg}$ group, and $0.5 \%$ in the placebo group. Non-melanoma skin cancer occurred in 5 patients in the tofacitinib group and 1 patient in placebo group. In addition, 5 patients in the tofacitinib group experienced a cardiovascular event, and the incidence of an increase in lipid level was higher in the tofacitinib than placebo group. The mechanism by which tofacitinib induces dyslipidemia is unknown. Chronic inflammation does decrease lipid levels, ${ }^{27}$ therefore, to be thought that the anti-inflammatory effect of tofacitinib would consequently increase lipid levels. ${ }^{28}$

\section{COMPARATIVE EFFECTIVENESS OF ANTI-TNF- $\alpha$} ANTIBODY, VEDOLIZUMAB, AND TOFACITINIB

The effectiveness of anti-TNF- $\alpha$ antibody, vedolizumab, and tofacitinib has not been directly compared. Thus, there currently are no clinical guidelines to inform the selection of the most appropriate drug to use, especially for patients who are naïve to anti-TNF- $\alpha$ antibody therapy. The network meta-analysis provided the following surface under the cumulative ranking value regarding the efficacy of biologics in patients with no prior history of anti-TNF- $\alpha$ antibody therapy: IFX, $85 \%$; vedolizumab, $82 \%$; GLM, 58\%; tofacitinib, $43 \%$; and ADA, $31 \%{ }^{29}$ The associated rate of mucosal healing was as follows: IFX, 91\%; vedolizumab, $81 \%$; tofacitinib, $54 \%$; GLM, $41 \%$; and ADA, $32 \%$. In the evaluation of the effectiveness of ADA, vedolizumab and tofacitinib among patients who had a previous history of TNF- $\alpha$ antibody therapy, tofacitinib provided the highest efficacy for achieving both clinical remission and mucosal heal- 
ing. It is important to note, again, that a head-to-head comparison of each drug was not conducted, which limits the translation of these results to practice.

The comparative efficacy of vedolizumab and anti-TNF- $\alpha$ antibody therapy was reported in a retrospective study at the 13th Congress of the European Crohn's and Colitis Organisation. Both the clinical remission rate and the endoscopic curative rate, after 12 months from the initiation of therapy with each of the drugs, were compared. The clinical remission rate was $38 \%$ for the vedolizumab group, compared to $34 \%$ for the anti TNF- $\alpha$ antibody therapy group (HR, 1.27; 95\% CI, 0.911.78 ), with an endoscopic healing rate of $50 \%$ versus $41 \%$, respectively (HR, 1.67; 95\% CI, 1.13-2.47). Although vedolizumab was deemed to be superior to anti-TNF- $\alpha$ antibody, cases of clinical failure on anti-TNF- $\alpha$ antibody therapy were included in the analysis and, as such, the effectiveness of anti-TNF- $\alpha$ antibody may have been underestimated. Therefore, the superiority of vedolizumab, as shown this study, should be interpreted with caution. However, the findings from this study do support the use of vedolizumab for patients who do not achieve a satisfactory response with anti-TNF- $\alpha$ antibody therapy.

Currently, there is insufficient evidence for a preferential selection of vedolizumab over tofacitinib. Tofacitinib has been associated with shingles and other infectious diseases, GI perforation, cardiovascular events, and elevation of serum lipids. Therefore, caution with prescription of this drug is indicated. On the contrary, it is considered that there are few adverse events in vedolizumab.

Based on current evidence, anti-TNF- $\alpha$ antibody therapy and tofacitinib may be considered as first-line biologics for the treatment of patients with severe UC. Furthermore, as there is currently greater evidence regarding the efficacy and safety of anti-TNF- $\alpha$ antibody therapy, compared to tofacitinib, the use of anti-TNF- $\alpha$ antibody therapy may first be considered. Vedolizumab may be used in cases that do not need a prompt induction of disease remission and that should be give priority to safety. In addition, Vedolizumab may be useful for patients who are difficult to maintain after induction of remission.

\section{INDIGO NATURALIS}

Indigo naturalis is fabricated from plants, including Indigofera tinctoria and Strobilanthes cusia, and contains both indigo and indirubin. Indole compounds, such as indigo and indirubin, contains an indole ring structure. Indole compounds are ligands for the aryl hydrocarbon receptor $(\mathrm{AhR})^{30}$ and act on innate type 3 lymphoid cells, expressing AhR. Recent studies have shown that the AhR signaling pathway stimulates the production of interleukin-22, ${ }^{31,32}$ which supports a role for indigo naturalis in the mucosal healing process. ${ }^{33}$ Therefore, indigo naturalis could provide an effective AhR ligand for the treatment of $\mathrm{UC}^{34}$

Historically, indigo naturalis has been used for the treatment of various inflammatory diseases and dermatitis, in China, ${ }^{35,36}$ including UC. Few studies have evaluated the therapeutic effectiveness of indigo naturalis for the treatment of UC. Sugimoto et al. ${ }^{37}$ reported the effectiveness of indigo naturalis. In this study, clinical response rate of a daily dose of $2.0 \mathrm{~g}$ of indigo naturalis for 8 weeks for the treatment of moderate-to-severe UC were $65 \%$. Naganuma et al. ${ }^{38}$ performed a prospective, randomized, double blind, placebo controlled trial on the efficacy of indigo naturalis, using a daily dose of $0.5,1.0$, or 2.0 $\mathrm{g}$, for 8 weeks, with a placebo group. The clinical response rate for each of the groups was as follows: $13.6 \%$ in the placebo group; $69.6 \%$ in the $0.5 \mathrm{~g}$ group; $75.0 \%$ in the $1.0 \mathrm{~g}$ group; and $81.0 \%$ in the 2.0 g group $(P<0.001$ for all indigo naturalis compared to the placebo group). During the time of the study, however, a report was published describing the development of pulmonary artery hypertension (PAH) among several patients who had purchased indigo naturalis without participating in a study. Therefore, Naganuma et al. ${ }^{38}$ terminated their trial due to safety considerations.

Adverse effects of indigo naturalis have been reported, including hepatic disorder, headache, nausea, vomiting, abdominal pain, intussusception, non-specific enteritis, and $\mathrm{PAH}^{39}$ Several factors can contribute to PAH development, including growth factors, cytokines, metabolic signaling, elastase, protease, and serotonin. ${ }^{40}$ Serotonin is a metabolite of tryptophan, an AhR ligand. ${ }^{31}$ As indigo is an AhR ligand, than administration of indigo naturalis might increase serotonin expression via the metabolic pathway of tryptophan. As serotonin acts on vascular smooth muscle cells, increases in serotonin levels would lead to the development of PAH. This postulated pathway, linking indigo naturalis to $\mathrm{PAH}$, remains to be verified (Fig. 1A).

Although the relationship between the dose of indigo naturalis and adverse effects is not clear, patients who develop PAH used indigo naturalis over a longer-term period. Adverse effects can be reversibly improved by discontinuing the use of indigo naturalis and/or appropriate therapeutic intervention. Of note, in patients who developed intussusception with the use of indigo naturalis, recurrence was noted with resumption 


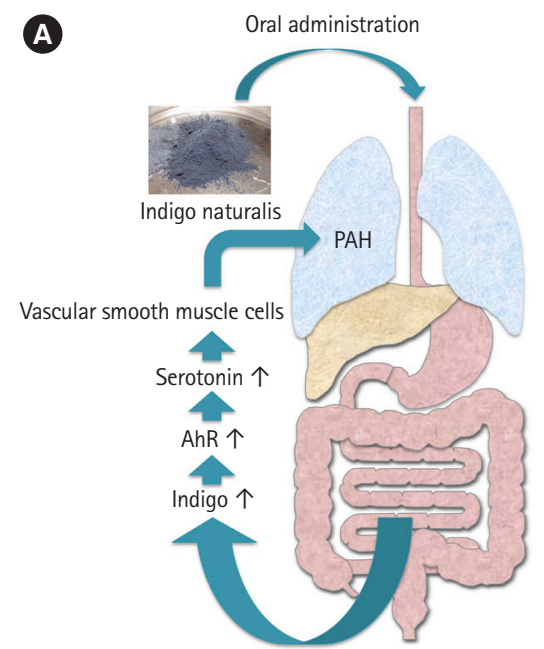

Absorption of indigo naturalis in the small intestine $\uparrow$
B

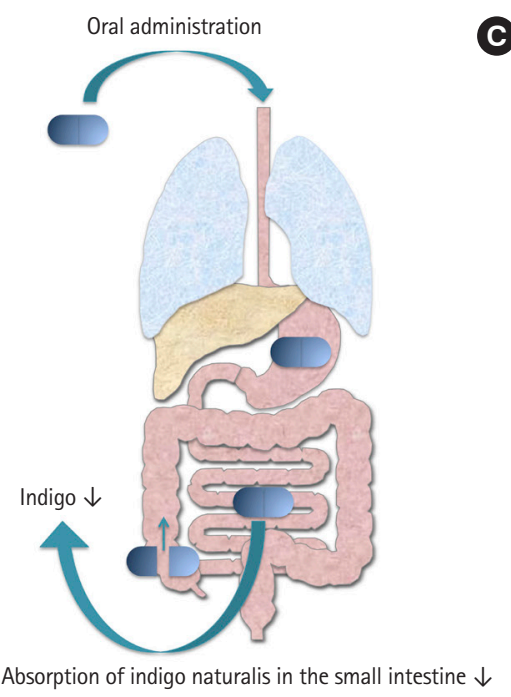

C No absorption of indigo naturalis in the small intestine

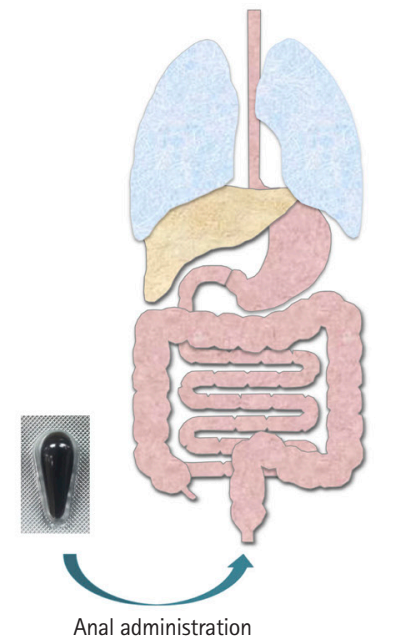

Fig. 1. Administration route of Indigo naturalis. (A) This is a hypothesis of the mechanism of pulmonary artery hypertension (PAH) onset by Indigo naturalis administration. Administration of Indigo naturalis increases the concentration of Indigo in the blood. Indigo acts as an aryl hydrocarbon receptor (AhR) ligand, resulting in an increase in serotonin concentration, a metabolite of tryptophan. Serotonin causes PAH. (B, C) shows an ingenuity of administration method to reduce side effects of Indigo naturalis. (B) The capsules that collapse in the large intestine decrease absorption of Indigo naturalis in the small intestine. (C) Suppository is administered without going through the small intestine.

of the use of indigo naturalis. ${ }^{41}$ Therefore, it is possible that some indigo naturalis-induced adverse effects could be recurrent.

Currently, as the safety of indigo naturalis has not been clearly established, it should be carefully used in practice, despite its known efficacy. Indigo naturalis should carefully be used for the treatment of patients with UC in whom induction of remission cannot be achieved with the use of corticosteroids, anti-TNF- $\alpha$ antibody therapy or calcineurin inhibitor. Considering that no advantage was found for a dose of indigo naturalis of $2.0 \mathrm{~g}$, compared to $1.0 \mathrm{~g}$, a dose of indigo naturalis of $\leq 1.0$ g per day should be used for the treatment of UC, and its use should be limited to 8 weeks to avoid side effects, such as PAH. Moreover, when using indigo naturalis, blood check tests, electrocardiogram, and echocardiogram assessments, among other assessment, should be regularly performed for early identification of adverse effects.

Before indigo naturalis can be used for UC treatment, its mechanisms of action will need to be concretely defined and its active components identified, and strategies to lower the risk of adverse events defined. In our lab, we are currently attempting to reduce the risk of adverse effects by suppressing absorption of indigo naturalis in the small intestine. To achieve this goal, we are attempting to develop indigo naturalis suppositories and capsules that collapse to deliver the dose of in- digo naturalis in the large intestine (Fig. 1B and C). We are also planning studies to elucidate how indigo, which is an AhR ligand contained in indigo naturalis, exerts its therapeutic effects on various immune cells, the intestinal epithelium and intestinal bacteria. There is also a need to link our results to drug discovery.

\section{FECAL MICROBIOTA TRANSPLANTATION}

FMT is a unique treatment that specifically improves intestinal microbiota. FMT gained much attention due to its effectiveness in the treatment of Clostridium difficile infection, described in 2013. ${ }^{42}$ Various studies have reported the intestinal microbiota change among patients with IBD. In fecal or intestinal epithelium of UC patients, it has been shown a lower relative level of the Firmicutes genus, such as Clostridium cluster IV and XIVa, with a relative higher proportion of the Bacteroidetes and Proteobacteria genus. ${ }^{43}$ However, it is unclear whether these changes in intestinal bacterial are the cause or the result of UC. The effectiveness of FMT for the treatment of UC has been evaluated in different randomized controlled trials. Moayyedi et al. ${ }^{44}$ reported a significantly higher remission rate of a 7-week period in the FMT (24\%) than the placebo (5\%) group. By comparison, Rossen et al. ${ }^{45}$ did not identify a therapeutic 
effectiveness of a 12-week period of FMT therapy, with a remission rate of $30 \%$ compared to $20 \%$ in the placebo group. Paramsothy et al. ${ }^{46}$ reported a therapeutic efficacy, providing FMT therapy 5 days per week for 8 weeks, with a remission rate of $27 \%$ compared to $8 \%$ in the placebo group $(P=0.021$ and $P=0.021$ ).

Although the effectiveness of FMT for the treatment of UC has been reported in a meta-analysis, ${ }^{47}$ the optimal protocol for administration (trans-nasally or trans-anal administration, with or without pretreatment antibiotic therapy, and donor eligibility) have remained not to be defined yet.

\section{FUTURE TASKS}

Although various treatments for IBD have been developed, there is currently insufficient evidence to inform the selection between established and novel treatments. As such, emerging treatments will continue to complicate the clinical management of UC. Furthermore, as treatment options increase, there is concern that patients will favor internal medicine approaches to treatment, which could delay surgical treatment, which can provide a curative effect. Increasingly, there will be a need to fully understand the mechanisms of action of the different therapeutic strategies, and to develop guidelines for treatment selection based on patient-specific characteristics.

\section{FINANCIAL SUPPORT}

The authors received no financial support for the research, authorship, and/or publication of this article.

\section{CONFLICT OF INTEREST}

No potential conflict of interest relevant to this article was reported.

\section{ACKNOWLEDGEMENTS}

We thank the present and past members of the Keio IBD Group for their continued support.

\section{AUTHOR CONTRIBUTION}

Fukuda T, Naganuma M, and Kanai T contributed to the drafting of the article, and contributed to critical revision of the article for important intellectual content. All the authors approved the final draft of the article.

\section{REFERENCES}

1. Ungaro R, Mehandru S, Allen PB, Peyrin-Biroulet L, Colombel JF. Ulcerative colitis. Lancet 2017;389:1756-1770.

2. Faubion WA Jr, Loftus EV Jr, Harmsen WS, Zinsmeister AR, Sandborn WJ. The natural history of corticosteroid therapy for inflammatory bowel disease: a population-based study. Gastroenterology 2001;121:255-260.

3. Palladino MA, Bahjat FR, Theodorakis EA, Moldawer LL. AntiTNF-alpha therapies: the next generation. Nat Rev Drug Discov 2003;2:736-746.

4. Rutgeerts P, Sandborn WJ, Feagan BG, et al. Infliximab for induction and maintenance therapy for ulcerative colitis. N Engl J Med 2005;353:2462-2476.

5. Adalimumab in the treatment of moderate-to-severe ulcerative colitis: ULTRA 2 trial results. Gastroenterol Hepatol (N Y) 2013;9:317-320.

6. Sandborn WJ, Feagan BG, Marano C, et al. Subcutaneous golimumab induces clinical response and remission in patients with moderate-to-severe ulcerative colitis. Gastroenterology 2014;146:85-95.

7. Sandborn WJ, Feagan BG, Marano C, et al. Subcutaneous golimumab maintains clinical response in patients with moderate-to-severe ulcerative colitis. Gastroenterology 2014;146:96109.el.

8. Gisbert JP, Panés J. Loss of response and requirement of infliximab dose intensification in Crohn's disease: a review. Am J Gastroenterol 2009;104:760-767.

9. Nanda KS, Cheifetz AS, Moss AC. Impact of antibodies to infliximab on clinical outcomes and serum infliximab levels in patients with inflammatory bowel disease (IBD): a meta-analysis. Am J Gastroenterol 2013;108:40-47.

10. Sofia MA, Rubin DT. Current approaches for optimizing the benefit of biologic therapy in ulcerative colitis. Therap Adv Gastroenterol 2016;9:548-559.

11. Vande Casteele N, Ferrante M, Van Assche G, et al. Trough concentrations of infliximab guide dosing for patients with inflammatory bowel disease. Gastroenterology 2015;148:13201329.e3.

12. Cesarini M, Katsanos K, Papamichael K, et al. Dose optimization is effective in ulcerative colitis patients losing response to infliximab: a collaborative multicentre retrospective study. Dig Liver Dis 2014;46:135-139.

13. Colombel JF, Panaccione R, Bossuyt P, et al. Effect of tight con- 
trol management on Crohn's disease (CALM): a multicentre, randomised, controlled phase 3 trial. Lancet 2018;390:27792789.

14. Imaeda H, Takahashi K, Fujimoto T, et al. Clinical utility of newly developed immunoassays for serum concentrations of adalimumab and anti-adalimumab antibodies in patients with Crohn's disease. J Gastroenterol 2014;49:100-109.

15. Imaeda H, Andoh A, Fujiyama Y. Development of a new immunoassay for the accurate determination of anti-infliximab antibodies in inflammatory bowel disease. J Gastroenterol 2012;47:136-143.

16. Hanauer SB, Wagner CL, Bala M, et al. Incidence and importance of antibody responses to infliximab after maintenance or episodic treatment in Crohn's disease. Clin Gastroenterol Hepatol 2004;2:542-553.

17. Panccione R, Ghosh S, Middleton S, et al. Infliximab, azathioprine, or infliximab + azathioprine for treatment of moderate to severe ulcerative colitis: the UC success trial. Gastroenterology 2011;140:S-134.

18. Ley K, Rivera-Nieves J, Sandborn WJ, Shattil S. Integrin-based therapeutics: biological basis, clinical use and new drugs. Nat Rev Drug Discov 2016;15:173-183.

19. Feagan BG, Rutgeerts P, Sands BE, et al. Vedolizumab as induction and maintenance therapy for ulcerative colitis. N Engl J Med 2013;369:699-710.

20. Engel T, Ungar B, Yung DE, Ben-Horin S, Eliakim R, Kopylov U. Vedolizumab in IBD-lessons from real-world experience: a systematic review and pooled analysis. J Crohns Colitis 2018; 12:245-257.

21. Kopylov U, Avni-Biron I, Ron Y, et al. Effectiveness and safety of vedolizumab for maintenance treatment in inflammatory bowel disease: the Israeli real world experience. Dig Liver Dis 2019;51:68-74

22. Amiot A, Grimaud JC, Peyrin-Biroulet L, et al. Effectiveness and safety of vedolizumab induction therapy for patients with inflammatory bowel disease. Clin Gastroenterol Hepatol 2016; 14:1593-1601.e2.

23. Christensen B, Gibson P, Micic D, et al. Safety and efficacy of combination treatment with calcineurin inhibitors and vedolizumab in patients with refractory inflammatory bowel disease. Clin Gastroenterol Hepatol. [published online ahead of print May 8, 2018]. https://doi.org/10.1016/j.cgh.2018.04.060.

24. Loftus EV Jr, Colombel JF, Feagan BG, et al. Long-term efficacy of vedolizumab for ulcerative colitis. J Crohns Colitis 2017;11: 400-411.

25. Clark JD, Flanagan ME, Telliez JB. Discovery and development of Janus kinase (JAK) inhibitors for inflammatory diseases. J Med Chem 2014;57:5023-5038.

26. Sandborn WJ, Su C, Sands BE, et al. Tofacitinib as induction and maintenance therapy for ulcerative colitis. N Engl J Med 2017;376:1723-1736.

27. Choy E, Sattar N. Interpreting lipid levels in the context of highgrade inflammatory states with a focus on rheumatoid arthritis: a challenge to conventional cardiovascular risk actions. Ann Rheum Dis 2009;68:460-469.

28. Wu JJ, Strober BE, Hansen PR, et al. Effects of tofacitinib on cardiovascular risk factors and cardiovascular outcomes based on phase III and long-term extension data in patients with plaque psoriasis. J Am Acad Dermatol 2016;75:897-905.

29. Vickers AD, Ainsworth C, Mody R, et al. Systematic review with network meta-analysis: comparative efficacy of biologics in the treatment of moderately to severely active ulcerative colitis. PLoS One 2016;11:e0165435.

30. Adachi J, Mori Y, Matsui S, et al. Indirubin and indigo are potent aryl hydrocarbon receptor ligands present in human urine. J Biol Chem 2001;276:31475-31478.

31. Zelante T, Iannitti RG, Cunha C, et al. Tryptophan catabolites from microbiota engage aryl hydrocarbon receptor and balance mucosal reactivity via interleukin-22. Immunity 2013;39: 372-385.

32. Qiu J, Guo X, Chen ZM, et al. Group 3 innate lymphoid cells inhibit T-cell-mediated intestinal inflammation through aryl hydrocarbon receptor signaling and regulation of microflora. Immunity 2013;39:386-399.

33. Kawai S, Iijima H, Shinzaki S, et al. Indigo naturalis ameliorates murine dextran sodium sulfate-induced colitis via aryl hydrocarbon receptor activation. J Gastroenterol 2017;52:904-919.

34. Sugimoto S, Naganuma M, Kanai T. Indole compounds may be promising medicines for ulcerative colitis. J Gastroenterol 2016;51:853-861.

35. Lin YK, Wong WR, Chang YC, et al. The efficacy and safety of topically applied indigo naturalis ointment in patients with plaque-type psoriasis. Dermatology 2007;214:155-161.

36. Deng S, May BH, Zhang AL, Lu C, Xue CC. Plant extracts for the topical management of psoriasis: a systematic review and meta-analysis. Br J Dermatol 2013;169:769-782.

37. Sugimoto S, Naganuma M, Kiyohara H, et al. Clinical efficacy and safety of oral Qing-Dai in patients with ulcerative colitis: a single-center open-label prospective study. Digestion 2016;93: 193-201.

38. Naganuma M, Sugimoto S, Mitsuyama K, et al. Efficacy of indigo naturalis in a multicenter randomized controlled trial of 
patients with ulcerative colitis. Gastroenterology 2018;154:935947.

39. Nishio M, Hirooka K, Doi Y. Chinese herbal drug natural indigo may cause pulmonary artery hypertension. Eur Heart J 2016;37:1992.

40. Schermuly RT, Ghofrani HA, Wilkins MR, Grimminger F. Mechanisms of disease: pulmonary arterial hypertension. Nat Rev Cardiol 2011;8:443-455.

41. Kondo S, Araki T, Okita Y, et al. Colitis with wall thickening and edematous changes during oral administration of the powdered form of Qing-Dai in patients with ulcerative colitis: a report of two cases. Clin J Gastroenterol 2018;11:268-272.

42. Razik R, Rumman A, Bahreini Z, McGeer A, Nguyen GC. Recurrence of Clostridium difficile infection in patients with inflammatory bowel disease: the RECIDIVISM study. Am J Gastroenterol 2016;111:1141-1146.

43. Nagao-Kitamoto H, Kamada N. Host-microbial cross-talk in inflammatory bowel disease. Immune Netw 2017;17:1-12.

44. Moayyedi P, Surette MG, Kim PT, et al. Fecal microbiota transplantation induces remission in patients with active ulcerative colitis in a randomized controlled trial. Gastroenterology 2015; 149:102-109.e6.

45. Rossen NG, Fuentes S, van der Spek MJ, et al. Findings from a randomized controlled trial of fecal transplantation for patients with ulcerative colitis. Gastroenterology 2015;149:110-118.e4.

46. Paramsothy S, Kamm MA, Kaakoush NO, et al. Multidonor intensive faecal microbiota transplantation for active ulcerative colitis: a randomised placebo-controlled trial. Lancet 2017; 389:1218-1228.

47. Costello SP, Soo W, Bryant RV, Jairath V, Hart AL, Andrews JM. Systematic review with meta-analysis: faecal microbiota transplantation for the induction of remission for active ulcerative colitis. Aliment Pharmacol Ther 2017;46:213-224. 